\title{
FLUIDEZ EN EL TRAZADO MANUAL Y COMPOSICIÓN ESCRITA. ESTUDIO EXPLORATORIO CON NIÑOS ARGENTINOS AL FINALIZAR TERCER GRADO
}

\author{
HANDWRITING FLUENCY AND WRITING COMPOSITION SKILLS. EXPLORATORY STUDY WITH \\ ARGENTINE CHILDREN OF THIRD GRADE
}

\author{
FABIÁN YAUSAZ*
}
*Profesor de Enseñanza Primaria, Licenciado en Psicología, MA en Enseñanza de la Lengua y la Literatura y Doctor en Letras con orientación en Lingüística. Miembro del Departamento de Investigación del Instituto Superior San José. E-Mail: fyausaz@yahoo.com.ar Barrio San Jerónimo, 60 Viviendas, Grupo 1, Mz. 9, Casa 12, (3400) Corrientes. República Argentina.
El autor agradece a los técnicos superiores en Psicología Cristhian Diego Fernández, María Agustina Cardozo y Brenda Natalí Ramírez su participación en la recolección de datos.

\section{RESUMEN}

En el estudio que se informa se analizó la fluidez del trazado manual en un grupo de niños argentinos que eran alumnos de tercer grado. Se pretendió, por un lado comparar las medidas de velocidad de copia con las consignadas por un estudio de referencia y por otro, explorar la relación entre el comportamiento de esta variable y el uso de la escritura en contexto de redacción. Este trabajo se alinea con la literatura que indaga el comportamiento de la memoria operativa durante la escritura. Tales estudios verificaron que la falta de fluidez en los procesos de transcripción restringe la ejecución de los procesos de composición escrita.

Participaron 122 niños de tercer grado de distintas escuelas que fueron evaluados a través de una prueba de copia y una narración escrita de cuento. Algunos resultados se alinearon con la literatura: la velocidad media de copia resultó próxima a la detectada en el estudio de referencia (Graham, Weintraub \& Berninger, 1998) y las niñas copiaron significativamente más rápido que los niños. Por otra parte, se verificó una variación entre las escuelas que fue vinculada con la influencia que ejercen las distintas modalidades de instrucción en el desarrollo de la fluidez. Por último, se encontraron correlaciones significativas entre la velocidad de copia y la precisión de la representación de las palabras en las narraciones. Con este resultado se interpretó la influencia que ejerce la fluidez en el acceso a representaciones ortográficas de palabras en actividades de escritura de distinto nivel de complejidad. También se consignan las implicaciones pedagógicas del estudio realizado.

Palabras clave: Fluidez del trazado manual; Composición escrita; Niños de tercer grado; Representación ortográfica de palabras; Memoria operativa.

\section{ABSTRACT}

The present study analyzes the handwriting fluency in a group of children who finished third grade of elementary education in Argentina. This work aims, on the one hand, to compare fluency level of these children, with a sample taken from other reference study; and on the other hand, to 
explore the relationship between handwriting fluency and measures of quality of writing composition. Consistently with the literature that characterized the function of working memory (WM) during writing (Berninger \& Swamson, 1996; Kellogg, 1996; McCutchen, 1996, 2000) the present work considers that the lack of proficiency in handwriting severely limits composition processes. In fact, if the writer has to focus his or her attention during composing to mechanical demands, such as think in the particular form of the letter, he or she may forget ideas or plans already held in WM (Graham \& Weintraub, 1996).

Children of third grade were chosen because literature in this field indicates that pupils of this level should reach a basic degree of mastery in handwriting processes. Also because the national language curriculum at the primary-school level in Argentina, in contrast with the policies adopted in most educational systems in the world, has relegated the development of transcription processes (handwriting, spelling, phonological awareness, orthographic knowledge) to a tangential or non-existent position.

One hundred and twenty two children of different SES were assessed. All of them were selected from a total of six schools in the cities of Corrientes and Resistencia in Argentina. Children were evaluated through two tests: a copying text and story writing. The first one estimated handwriting speed (with letters per minute measure), whilst the second one assessed different characteristics of text quality (length, word-segmentation errors and orthographic mistakes).

The comparison of speed of copy against a sample of United States (Graham, Weintraub \& Berninger, 1998) showed that the average results obtained by children of this study, reach similar level of fluency of the participants of that reference sample. In the same vein than specialized literature, it was found that girls copied faster than boys (Berninger \& Fuller, 1992). In addition, differences associated with the school of attendance were found. This result was interpreted as a remark of the importance that instructional factor has in the development of handwriting fluency.

An analysis of correlation showed that it could not be detected any associations between speed of copy and length of the story composed. This was a surprising result since it was supposed that differences of fluency among schools would be associated with differences of text length. It was documented that differences in handwriting fluency predict the amount and quality of children writing (Jones \& Christensen, 1990). The nonsignificant correlation was related to the low quality of most of the texts written by children. Bourdin and Fayol (2002) specified that when the task of writing is too easy, it is difficult to detect the influence of transcription on composition processes.

Finally, significant associations were detected between fluency and problems of word representation (spelling and word segmentation) in the texts composed. These results showed that the lack of fluency in the access to the orthographic representations of words is related to both tasks of writing above mentioned. When this kind of representation is not easily available, the writer uses anti-economic strategies of word writing, like phonological analysis. It was observed that these processes reduced the speed of handwriting in the copy task and caused orthographic mistakes in the story writing. An extra evidence of this situation was observed during the copy test. In this task, children who copied particularly slow, whispered a phonological analysis in most of the words that they were copying. Although several researches that examined the performance of children with writing difficulties, reported lack of writing continuity during the copy, there were not found references in that studies to this process of phonological analysis.

Pedagogical implications of these findings were also considered through the present study.

Keywords: Handwriting fluency; Writing composition; Children of third grade; Orthographic word representation; Working memory.

\section{INTRODUCCIÓN}

Durante los últimos 25 años un conjunto de estudios cognitivos especializados en los procesos de composición escrita se ocupó de analizar las restricciones que la memoria operativa (MO) impone al proceso de re- 
dacción (Kellogg, 1996; Mc Cutchen, 1996, 2000). En este sentido, Rodríguez y colaboradores (2010) reseñan los modelos de redacción propuestos por Hayes (2006), Kellogg (1996) y Butterfield, Hacker y Albertson (1996). La reseña destaca que la MO desempeña un papel crucial en la arquitectura de estos tres modelos porque este dispositivo da cuenta satisfactoriamente de las múltiples tareas simultáneas que ejecuta el escritor. Además los autores consignan que este constructo ha sido testeado empíricamente y confluye con los modelos pare el TDAH en la infancia.

Desde mediados de la década del 90 se compararon las diferentes exigencias cognitivas de la redacción y la producción oral y ello demostró que la transcripción restringe en mayor medida que la articulación del habla la asignación de recursos de $\mathrm{MO}$ a los procesos de composición tanto en niños (Berninger \& Swanson, 1994; Borzone \& Diuk, 2003; Bourdin \& Fayol, 1994; Grabowski, 2005; Graham, Harris \& Fink, 2000; Olive, Beavuais, C. \& Beauvais, L., 2009; Sánchez Abchi, Borzone \& Diuk, 2007) como en adultos (Bourdin \& Fayol, 2002).

Berninger y Swanson (1994) propusieron un modelo de redacción en el que discriminan los procesos que permiten la organización discursiva del escrito (procesos de generación textual) y los que permiten su realización física (transcripción). Los trabajos especializados (Bourdin \& Fayol, 1994; Grabowsky, 2005; Graham, Harris \& Fink, 2000) demostraron que las restricciones de recursos de MO que impone la transcripción a la composición son mayores en los escritores aprendices y que disminuyen con el aumento en la automaticidad de ejecución del trazado manual.

Berninger y Graham (1998) consideran que las acciones de trazado poco fluidas pueden interferir la ejecución simultánea de procesos de composición escrita. En efecto, la falta de fluidez en la ejecución de los programas motores incrementa la demanda de recursos de MO (Jones \& Christensen, 1999) y puede ocasionar el olvido de las ideas instanciadas en la MO antes de transcribirlas, el cansancio, la pérdida de interés en la tarea o el abandono súbito de la misma (Graham \& Wentraub, 1996).

En el marco de estos estudios cobró relevancia la estimación de la fluidez y la precisión o legibilidad del trazado. No obstante, Medwell y Wray (2008) opinan que en la última década la investigación sobre el trazado manual ha resultado escasa. De hecho, los trabajos incluidos en los volúmenes editados por Graham y Fitzgerald (2006) y Troia (2009) destacan que uno de los antecedentes más importantes en este campo es el meta-estudio emprendido por Graham y Weintraub (1996) en el cual fueron relevadas investigaciones realizadas entre los años 1980 y 1994.

La literatura especializada propone un constructo denominado programa motor de trazado (Graham \& Weintraub, 1996). Dicho programa es concebido como la representación mental de los movimientos necesarios para la realización de los rasgos escritos. Este programa se distingue del concepto tradicional de aprestamiento (Condemarín, Chadwick \& Milicic, 1994) entendido como acciones de enseñanza y práctica que favorecen la maduración de procesos psicomotores. El programa motor de trazado, por su parte, es concebido como la resultante de la interacción entre procesos madurativos y acciones instruccionales (o acciones de aprestamiento). En este sentido, Berninger y Winn (2006) opinan que la instrucción adecuada permite integrar secuencias de movimientos específicos y planes de naturaleza lingüística.

Berninger condujo un programa de investigación focalizado en el desarrollo de habilidades de escritura denominado lenguaje a través de la mano. Estos trabajos articularon resultados provenientes de investigaciones evolutivas, didácticas y evidencias neurofisiológicas (ver Berninger \& Graham, 1998). Basándose en un enfoque funcional del sistema mente-cerebro (Luria, 1973), esta perspectiva supone que el lenguaje a través de la mano es un sistema lingüístico que posee una singular organización mental, una trayectoria evolutiva específica 
y que, a lo largo del desarrollo, interactúa de manera diferente con los otros tres sistemas lingüísticos (lenguaje a través del oído, de la boca y de los ojos)

Con el objeto de testear las relaciones entre el lenguaje a través del ojo y de la mano, uno de los experimentos presentados por Berninger, Abbott, R., Abbott, S., Graham y Richards (2002) contrastó pruebas de copia y de lectura de palabras realizadas por 600 niños de escuela primaria. El análisis de regresión múltiple (path analysis) muestra que el reconocimiento de palabras ejerce una influencia significativa sobre la fluidez de transcripción pero que la relación inversa sólo se verifica en niños de segundo grado. Esto implica que la mayor disponibilidad de representaciones de palabras suficientemente discriminadas en la memoria a largo plazo influye sobre la copia más veloz, pero que la copia fluida no necesariamente ayuda a configurar representaciones en el léxico mental.

Subyace a estos resultados que los sistemas de lenguaje a través de la mano y del ojo se interrelacionan en la configuración de representaciones léxicas. Los trabajos que se ocuparon del aprendizaje inicial de la lectura (Ehri, 1992; Perfetti, 1992) suponen que los procesos de recodificación fonológica interactúan con la información léxica (la sucesión de letras que permite identificar una palabra) y que esta interacción favorece la configuración de representaciones ortográficas de palabras. Estas representaciones amalgaman información semántica, fonológica y ortográfica. Basándose en la evidencia empírica reseñada anteriormente, Berninger, Garcia y Abbot (2009) proponen incorporar una representación del trazado a estas entradas léxicas.

En Argentina algunos trabajos (Sánchez Abchi, Diuk, Borzone \& Ferroni, 2009; Signorini \& Borzone, 2003) abordaron la especificidad de los procesos de aprendizaje de la escritura a través de dictados de palabras. Estas investigaciones exploraron la interacción entre los conocimientos fonológicos y los ortográficos, pero no analizaron la influencia que podría ejercer la fluidez de trazado en las primeras etapas del aprendizaje de la escritura.

Con relación a las mediciones de habilidades de escritura en Español recientemente han sido estandarizadas las baterías PROESC (Cuetos, Ramos \& Ruano, 2002), TALE-2000 (Toro, Cervera \& Urío, 2000) y LEE (Defior Citoler. Fonseca \& Gottheil, 2006). Específicamente los baremos de LEE incluyeron niños argentinos de primero a cuarto grado. No obstante, con relación al presente artículo sólo el test TALE-2000 incorpora dos pruebas de copia, pero solamente mide variables de legibilidad sin considerar la fluidez del trazado.

Por su parte, estudios que abordaron específicamente el trazado manual se basaron en los resultados de pruebas de copia. HamstraBletz y Blote (1990) siguieron a 127 niños holandeses desde segundo hasta sexto grado. Los autores constataron que, mientras estos niños recibían una instrucción escolar en trazado (segundo y tercer grado), tanto la fluidez como la legibilidad evolucionaban notablemente; entre cuarto y sexto grado (cuando la instrucción era interrumpida) la fluidez continuó evolucionando pero el trazo adoptó rasgos más personales y la legibilidad disminuyó.

Con respecto a la velocidad de copia, existe consenso en estimarla a través de la medición de letras por minuto. Así y todo, los trabajos no presentan una tendencia normal homogénea. Graham, Weintraub y Berninger (1998), por ejemplo, reseñan cuatro estudios en los cuales se observa en niños de tercer grado, una variación entre 25 y 39 letras por minuto, en tanto que el propio trabajo detectó una media igual a 47.3.

Por un lado, esta variación se vincula con el tipo de instrucción escolar que recibieron los participantes de los distintos estudios y, por otro, con la metodología de recolección de datos adoptada. Por ejemplo, HamstraBeltz y Blote (1990) y Overveld y Hulstijn (2011) solicitaron a los niños que copiaran un texto durante 5 minutos, Graham y colaboradores (1998) que lo hicieran durante un minuto y medio, mientras que Condemarín y Chadwick (1986) solicitaban la copia de una 
misma oración la mayor cantidad de veces posible durante un minuto.

Trabajos que se han ocupado de los niños con problemas de aprendizaje de la escritura o disgrafía (Graham \& Weintraub, 1996; Hamstra-Bletz \& Blote, 1993; Overveld \& Hulstijn, 2011) destacan la menor velocidad media de copia en esta población. Entre otras cuestiones, estas investigaciones detectaron una mayor frecuencia de pausas durante la tarea. A resultados similares arribaron los trabajos que midieron la velocidad solicitando que los sujetos copiaran con plantillas y lápices digitales (Rosenblum, Parush \& Weiss, 2001). En este sentido, Rosemblum y colaboradores (2003, ver Rosemblum, Weiss \& Parush, 2004) consignaron que los niños con disgrafía mantenían el lápiz en el aire significativamente más tiempo que los niños de un grupo control.

Berninger y Richards (2002) puntualizan que resulta prioritario desarrollar fluidez en los procesos de transcripción durante los tres primeros años de escolaridad. Para ello se incorporan acciones específicas de instrucción. Esta parece ser la tendencia que se verifica en sistemas educativos como el francés (Olive et al., 2009), el chileno (Condemarín \& Chadwick, 1986), el norteamericano (Berninger \& Richards, 2002), el inglés (Medwell \& Wray, 2008) o el holandés (Hamstra-Bletz \& Blote, 1990).

Los diseños curriculares argentinos adoptaron desde fines de la década del 80 dos medidas que no encuentran correlato con las decisiones políticas de otros países ni son respaldadas por sólida evidencia empírica, a saber: iniciar la enseñanza de la escritura (y de la lectura) utilizando sólo letra imprenta mayúscula y no proponer durante la primera escolaridad un programa sistemático para el desarrollo de las habilidades de trazado manual. El supuesto teórico que fundamentó esta decisión fue que la inclusión recurrente de los niños en situaciones en las cuales se escribiera funcionalmente bastaba para que los niños ejecutaran cada vez más eficientemente los procesos de trazado. Es probable que, debido a esta decisión didáctica, no se hayan encontrado traba- jos realizados en el país que analicen la evolución del trazado manual.

Por lo tanto, el objetivo del estudio que se informa fue explorar las relaciones entre la fluidez de trazado manual e indicadores de uso de la escritura en contexto de redacción en niños argentinos que finalizaron tercer grado. Se pretendió, por un lado, relevar medidas de fluidez de copia y contrastarlas con las obtenidas por un estudio de referencia. También se buscó sondear las relaciones entre la fluidez de trazado y algunos indicadores del uso de la escritura en contexto de redacción.

El estudio realizado se inscribe en una serie de trabajos que pretenden establecer medidas estandarizadas de escritura para la región del nordeste argentino.

En línea con el modelo de MO consignado anteriormente se presume que la fluidez del trazado manual permitirá liberar recursos de procesamiento que podrán ser asignados a la composición.

\section{Metodología}

\section{SUJETOS}

Participaron 122 niños (63 mujeres y 59 varones), de los cuales, 100 niños contaban con la edad prevista por el sistema educativo argentino para ese ciclo escolar (edad promedio 8.07 , extremos 8.05 y 8.10 ) y 22 con sobre-edad (promedio 11.02, extremos 10.01 y 12). Para configurar esta población el criterio de inclusión adoptado fue que los niños hubieran cursado la escolaridad íntegra en el mismo establecimiento.

Dado que el estudio pretende establecer medidas estandarizadas de escritura para la región del nordeste argentino, se conformó una muestra que fuera heterogénea con respecto a la procedencia escolar de los niños. Fueron evaluados seis grupos completos de alumnos de seis escuelas de las ciudades Corrientes Capital y Resistencia (Chaco). Dos de estas escuelas eran privadas y atendían mayoritariamente niños de procedencia social media alta y alta; otras dos eran pú- 
blicas, estaban ubicadas dentro del radio céntrico de ambas ciudades y su población era mayoritariamente de niños de clase media y media-baja, en tanto que las otras dos, eran escuelas públicas y sus alumnos provenían de barrios habitados por familias que viven en contextos de extrema pobreza.

En una escuela el investigador responsable participó de una reunión en la cual informó a los padres sobre los objetivos del trabajo y solicitó el consentimiento informado para realizar la recolección. Los directivos de las otras cinco escuelas consideraron que las pruebas no diferían de las tareas escolares cotidianas y, por este motivo, evaluaron que no era necesario solicitar el consentimiento de los padres.

\section{INSTRUMENTOS}

\section{PRUEBA DE COPIA DE TEXTO}

La prueba consiste en solicitar la copia de un texto breve durante un minuto y medio (Graham et al., 1998). Un entrevistador entrega a cada niño una ficha con el texto fuente y solicita la copia del mismo durante el tiempo establecido.

Antes de comenzar la prueba, el evaluador solicita que los niños realicen un comienzo y una detención de la copia a manera de prueba. Para determinar la fluidez de copia se estima el coeficiente de letras copiadas por minuto.

\section{PRUEBA de NARRACIÓN ESCRITA DE CUENTO}

Esta prueba consiste en la lectura en voz alta de un cuento breve y posteriormente se solicita la narración escrita.

Se trabajó con dos versiones de relatos folklóricos que ya habían sido utilizadas en estudios realizados en la región (Borzone \& Diuk, 2003; Yausaz, 2011); ambas tienen una extensión similar y su organización responde a la estructura de un episodio (Trabasso \& Rodkin, 1994). Se determinó de manera aleatoria la versión leída a los dis- tintos niños. En todas las narraciones escritas se estimaron los valores de las siguientes variables:

1.- Extensión de los textos: Es la cantidad de palabras que tienen los textos. Ante los errores de segmentación de palabras se adoptó como criterio la representación convencional que el niño hubiera producido si no hubiese existido el error. Por ejemplo $<$ en tonces $>$ (entonces) fue computada como una palabra, $<$ timporta $>$ (te importa) como dos.

2.- Cociente de errores de segmentación: Se contabilizaron las palabras no segmentadas convencionalmente (por ej. $<$ alado $>$ por $<$ al lado $>$ ) y este resultado fue dividido por el total de palabras del texto redactado por el niño.

3.- Nivel ortográfico global (NOG): En línea con Tuana (1980) esta medida equivale al porcentaje de palabras de los textos escritas correctamente. Para la estimación no fueron ponderados los errores de representación de rasgos suprasegmentales (acentos, diéresis).

Ambas pruebas fueron administradas en el mes de noviembre (cuando los niños finalizaban tercer grado) y estuvieron a cargo de cuatro evaluadores. La prueba de copia fue realizada en el salón de clases con el grupo total de niños y la narración escrita fue administrada en subgrupos de 3 ó 4 niños.

\section{ANÁLISIS DE LOS DATOS}

Para comparar el desempeño en la prueba de copia de los subgrupos se realizó un análisis de la variancia (ANOVA de un factor) teniendo en cuenta los siguientes factores: sexo, mano con la que escribían los niños, edad, procedencia social y escuela a la que asistieron. Para el análisis post-hoc se utilizaron las pruebas Tukey y Tamhane (en función de las presunciones a partir del test de homogeneidad de variancias). Como todas 
las variables fueron medidas en escalas de razón, el análisis de la correlación fue realizado a través de $r$ de Pearson.

\section{Resultados}

En principio se compararon las medidas de fluidez de copia obtenidas por los sujetos del presente estudio con las de niños norteamericanos evaluados con la misma metodología.

La Tabla 1 muestra que las medidas del grupo total y de los subgrupos por sexo resultaron próximas a los datos consignados por el estudio de referencia. En el subgrupo de niños con sobre-edad, en cambio, se detectó una velocidad aproximadamente un $50 \%$ más lenta que en el trabajo norteamericano (51.3 versus 84.74$)$.

Graham y Weintraub (1996) señalan que en la evolución de las habilidades de copia inciden tanto la maduración como la instrucción. Con relación a los resultados enunciados, el avance en el cursado de la escolaridad formal parece ser un factor que explica mejor que la edad cronológica, el diferente nivel de fluidez alcanzado por los niños mayores del estudio de referencia.

Para continuar explorando el comportamiento de esta variable se realizó la prueba ANOVA discriminada por los factores consignados relevantes por el estudio de referencia. Si bien la media de los niños con sobreedad fue superior a la obtenida por niños con la edad esperada para tercer grado, la diferencia no resultó estadísticamente significativa $(F=1.27 ; p>.05)$, tampoco se encontraron diferencias asociadas con la mano que utilizaban los niños para escribir $(F=.48$; $p>.05$ ). En línea con la literatura (Berninger \& Fuller, 1992), las niñas copiaron significativamente más rápido que los niños $(F=12.9 ; p<.000)$. Graham y Weintraub (1996) destacan que esta tendencia ha sido suficientemente documentada y puntualizan que la misma puede estar relacionada con factores madurativos y culturales.

La Tabla 2 presenta los resultados de fluidez de copia según escuela y procedencia social y muestra una importante variación en las medidas de fluidez entre las escuelas. Los alumnos de las escuelas 1,3 y 5 presentaron medias superiores al estudio de referencia, en tanto que en los otros tres grupos se verificaron medias inferiores. Esta variación no se corrobora si se discriminan los subgrupos por procedencia social. En efecto, la prueba ANOVA para el factor Escuela resultó significativa $(F=7.8 ; p<.000)$, en tanto para el factor Procedencia Social no arrojó resultados significativos. Se especula con que esta variación interescuelas se relaciona con el tipo de enseñanza impartida en cada una de ellas. No fue controlada en todas las escuelas la metodología de enseñanza de la escritura desarrollada durante los tres primeros años de escolaridad. No obstante, dos de las escuelas habían participado de un estudio anterior (Yausaz, 2011) y en estos casos se pudo obtener esta información.

Los niños de la escuela 5 habían recibido una enseñanza que abordaba en paralelo la transcripción y composición escrita (inspirada en los lineamientos de Borzone, Rosemberg, Diuk, Silvestri \& Plana, 2004 y Yausaz, 2005). Estos niños estaban habituados a destinar un tiempo semanal a las actividades de copia y de dictado en situaciones temporalmente próximas a propuestas de redacción. La media de fluidez de estos niños se ubica en el extremo superior de la muestra (57.4).

En tanto los alumnos de la escuela 4 habían participado durante los tres primeros grados de un programa nacional actualmente vigente en la República Argentina (MECyT, 2006). Dicho programa impulsa la escritura de textos de invención y no promueve la ejercitación descontextualizada de los procesos de transcripción. Los niños de esta escuela presentaron la media de fluidez más baja (27.4) de todos los grupos relevados. Esta medida es inferior a la consignada en el estudio de Graham y colaboradores (1998) para niños de segundo grado y similar a la hallada por Overveld y Hulstijn (2011) en niños de segundo grado que habían recibido un diagnóstico de disgrafía.

Para explorar la relación en el desempeño de los sujetos en las dos pruebas se realizó un 
análisis de correlación entre la velocidad de copia y las variables que medían la calidad de las narraciones. No se verificó una correlación significativa entre la velocidad de copia y la extensión de los textos narrados.

Se esperaba que la diferencia inter-escuelas consignada anteriormente se asociara con una variación en la extensión de los textos redactados. En efecto, en línea con Jones y Christensen (1999) se suponía que los mayores niveles de automatización permitirían liberar recursos de MO y asignarlos a la generación textual (aspecto que se evidenciaría en la redacción de textos más extensos), en tanto que los menores niveles de automatización se asociarían con la asignación de recursos de MO al control de la correcta ejecución del trazado. También se presuponía que los resultados del trabajo realizado se alinearían con los obtenidos por Borzone y Diuk (2003), allí fueron detectadas correlaciones medias entre el conocimiento ortográfico (medido a través de un dictado de palabras) y la extensión de textos. Ninguna de estas presunciones pudo ser verificada.

En cambio, la velocidad de copia correlacionó con el NOG y con los errores de segmentación $(r=.3, p<.001$ y $r=-.29$, $p<.001$ ), es decir que los niños que copiaron más rápido tendieron a escribir convencionalmente mayor cantidad de palabras en sus narraciones y cometieron menor cantidad de errores de segmentación.

Si se adopta un modelo del funcionamiento de la MO durante los procesos de ejecución (Kellogg, 1996) se puede conjeturar que durante la copia el niño primeramente observa el texto - modelo, luego retira la vista del mismo y retiene un fragmento en la agenda viso-espacial, posteriormente activa representaciones ortográficas de palabra de su léxico mental y las instancia en el bucle fonológico $(\mathrm{BF})$ para ejecutar la copia. $\mathrm{Si}$ el niño no cuenta con representaciones de este tipo o si las mismas no están suficientemente discriminadas, debe recurrir a procedimientos que ralentizan el proceso (subvocalizar secuencialmente los fonemas de las palabras o mirar el modelo con mayor frecuencia).
La dificultad de acceso a las representaciones de palabra también afecta la tarea de narración escrita. A continuación se analiza un ejemplo en este sentido (ver Figura 1).

La niña representa la palabra "ayudarla" como $<$ a chudarla $>$ en un renglón y como $<$ ayudarla $>$ en el siguiente. El error ortográfico $<\mathrm{ch}>$ en lugar de $<\mathrm{y}>$ fue detectado frecuentemente por Yausaz (2011) en niños correntinos, fue vinculado con la influencia de procesos fonológicos sobre la escritura ortográfica y no fue documentado por estudios realizados con niños de Buenos Aires. A diferencia del Español rioplatense, variedad en la que el fonema palatal aproximante $(/ \mathrm{y} / \mathrm{j} /)$ se realiza como fricativa postalveolar $\left(/ 3 / 0 / \int /\right)$, en la variedad no estándar del Español de Corrientes (Abadía de Quant, 2000) lo más frecuente es la realización como una africada sonora $(/ \mathrm{d} 3 /)$.

Supuestamente la niña formuló una frase ("pensó ayudarla") y la instanció en el BF para transcribirla. Se presume que el flujo cognitivo (Kellogg, 1996) se detuvo cuando la niña no pudo acceder a la representación ortográfica de "ayudarla". Ante esta situación el ejecutivo central cambió el foco atencional de la tarea, instanció la palabra en cuestión en el BF y destinó recursos de procesamiento al análisis secuencial de los fonemas. Como este análisis se llevó a cabo en el marco de una tarea de redacción, es probable que compitiera con otros procesos de ejecución simultánea por la disponibilidad de recursos de procesamiento de MO. Se interpreta que los errores en la escritura son un efecto de esta pugna, la niña escribió la palabra produciendo un error de segmentación y otro de representación fonológica: en lugar de la africada sonora propia de su variedad oral $(/ \mathrm{d} 3 /)$, representó una africada sorda $(/ \widehat{\mathrm{t}} /)$.

En el segundo renglón la niña representó la misma palabra de forma convencional, una posibilidad es que haya repetido el proceso de análisis secuencial y que este procedimiento haya producido una variación en la escritura y otra, es que el análisis fonológico emprendido la primera vez haya facilitado el acceso de la ruta ortográfica. En 
cualquiera de los dos casos, el proceso de análisis secuencial, además de detener el flujo de generación textual, produjo una variación en la representación de palabras.

En línea con los modelos que se ocuparon de la $\mathrm{MO}$ en niños que aprendían a escribir se interpretan las correlaciones consignadas en los siguientes términos: la asociación entre velocidad de copia y la escritura convencional de las palabras en las narraciones evidencia que ambas tareas se ven influenciadas por el grado de fluidez en el acceso a representaciones ortográficas de palabra. En la primera la dificultad en el acceso se asociaría con una ralentización de la copia, en tanto que en la segunda, con la representación imprecisa de las palabras.

Una evidencia extra que apoya estas interpretaciones es la frecuencia de la subvocalización. Durante la administración de las pruebas se observó que los niños que copiaban más lentamente subvocalizaban con mayor frecuencia las palabras del texto modelo. Si bien los estudios en los cuales participaron niños con problemas de aprendizaje de la escritura consignan una mayor lentitud en tareas de copia (Graham \& Weintraub, 1996; Rosemblum et al., 2001), no se encontraron alusiones al proceso de subvocalización. En términos generales estos trabajos se focalizan en los aspectos motores del trazado. En el apartado siguiente se establecerán comparaciones entre estos resultados y los obtenidos en dos estudios que en Argentina abordaron la relación entre lectura y prosodia (Borzone de Manrique \& Signorini, 1991, 2000) y que también analizaron el proceso de subvocalización.

Con el objeto de profundizar el análisis de la relación entre conocimientos ortográficos y fluidez de copia, la población fue dividida por el NOG detectado en las narraciones y luego se realizó un análisis de variancia entre ambos subgrupos. Como se esperaba, el subgrupo que alcanzó o superó la media de NOG consignada por Tuana (1980) para tercer grado copió significativamente más rápido que el que presentó un nivel inferior $(F=9.95 ; p<.01)$.

\section{Discusión}

En el estudio que se informa se exploraron las relaciones entre la fluidez en una prueba de copia y distintos indicadores del uso de la escritura en contextos de redacción. Dado que el sistema escolar argentino no promueve la ejercitación descontextualizada del trazado manual se especulaba que estos niños obtendrían medidas de fluidez sensiblemente inferiores a las alcanzadas por niños que reciben una instrucción de este tipo. Este presupuesto no se constató.

A partir de los resultados obtenidos podría concluirse que, para alcanzar un nivel de fluidez normal o esperable no es necesaria la práctica descontextualizada y basta con involucrar a los niños en situaciones de producción escrita. Sin embargo, la variación inter-escuelas detectada y la comparación del desempeño de los niños de las escuelas 4 y 5 presentados en el apartado anterior permite poner en duda esta afirmación.

La literatura especializada (Berninger \& Richards, 2002; Hamstra-Bletz \& Blote, 1993) reporta los beneficios de programas precoces que aborden de forma descontextualizada el trazado manual.

Estudios posteriores deberán determinar, cuánta ejercitación de este tipo es suficiente para que la mayor parte de la población escolar alcance, al finalizar el primer ciclo de la escolaridad obligatoria, un piso de fluidez en el trazado.

Por otra parte también sería beneficioso para la toma de decisiones educativas contar con muestras estandarizadas de fluidez realizadas en la República Argentina que permitieran estimar cuál es el intervalo considerado normal o esperable. Por esperable se entiende la ejecución del trazado con un nivel de fluidez que no entorpezca las tareas de composición que el sistema educativo considera prioritario abordar. Además, estudios de este tipo también permitirían determinar cuáles son los niveles de fluidez asociados con potenciales dificultades de aprendizaje de la escritura.

En Argentina, Fonseca (2009) encontró que los valores de ciertas pruebas de la escala 
LEE predecían de manera confiable las dificultades en lectura. Sería relevante para el sistema educativo contar con mediciones similares con respecto a la fluidez en la escritura.

Se esperaba encontrar una correlación entre fluidez y extensión de textos porque la literatura especializada (Berninger \& Graham, 1998; Graham, 1990; Jones \& Christensen, 1999) sugiere que el trazado excesivamente lento puede interferir la ejecución simultánea, por parte de la MO, de los procesos de generación textual. Con respecto a la inexistencia de esta correlación se esbozan dos conjeturas:

La primera se vincula con la sencillez de las narraciones recolectadas. Si bien la organización discursiva de las narraciones no fue objeto de análisis, la mayor parte de los escritos estaban compuestos por una sucesión de cláusulas yuxtapuestas o conectadas a través de la conjunción $/ y /$, menos del 10\% incluyó algún signo de puntuación, el tiempo medio que los niños invirtieron en narrar fue 5' 23". La extensión media de los relatos (50 palabras) fue similar a la obtenida por Borzone y Diuk (2003), quienes reportaron en aquellos textos dificultades discursivas de todo tipo.

Puede inferirse que los niños redactaron poniendo en funcionamiento procedimientos de 'decir el conocimiento' (Bereiter \& Scardamalia, 1987). En otras palabras, para mantener la tarea de redacción dentro de límites manejables, la mayor parte de la población activó en su memoria a largo plazo la información del texto-fuente y la 'dijo' sobre el papel sin atender a aspectos de cohesión o coherencia. La simplificación de la tarea de redacción dificultó observar el efecto que ejerció la variación de fluidez detectada sobre la extensión de los textos.

En este sentido, en el experimento de Bourdin y Fayol (2002) en el cual se compararon narraciones escritas y orales, a los sujetos adultos se les solicitó el recuerdo libre de palabras y su inclusión en una historia. Se verificó que también en los adultos la transcripción suponía mayor carga cognitiva que la articulación verbal. Los autores puntualizan que este resultado no había sido obser- vado en un experimento anterior en el que sólo se había solicitado el recuerdo libre de palabras (Bourdin \& Fayol, 1994).

Investigaciones posteriores deberán indagar si, en condiciones de recolección de datos que involucren a los niños de tercer grado en una tarea de redacción cognitivamente más exigente, se puede verificar la asociación entre fluidez de trazado y extensión de los textos.

La segunda conjetura a la que se aludía anteriormente es que en los niños que cursan este ciclo educativo, la fluidez se encuentre menos asociada que la legibilidad con la extensión de los textos. En efecto, la mayor parte de los estudios sobre trazado consignados en la Introducción se ocupó de la velocidad y de la calidad de copia o legibilidad. En el estudio realizado los errores de trazado no fueron tenidos en cuenta, entre otros motivos por la dificultad que supone ponderar objetivamente la legibilidad. En este sentido, Rosenblum y colaboradores (2004) destacan que ha sido difícil arribar a un consenso en la estimación de esta variable. Por este motivo tanto los trabajos de estos autores como el de Guinet y Kandel (2010) han propuesto utilizar medios digitales de recolección. Futuras investigaciones podrán indagar aspectos de la legibilidad del trazado y su relación con la extensión de los textos redactados por los niños. Este tipo de estudios también podría aportar evidencia empírica favorable o no, a la decisión de la República Argentina de comenzar la enseñanza de la escritura utilizando letra imprenta mayúscula.

Las correlaciones entre la velocidad de copia y las medidas ligadas a la representación precisa de palabras se alinean con los trabajos de Berninger y Richards (2002) y Berninger y colaboradores (2009) que conciben a la copia no sólo como la ejecución precisa de movimientos manuales, sino también como un proceso lingüístico ejecutado a través de un canal motor.

La detención y la subvocalización observada con mayor frecuencia en los niños que copiaban más lentamente es convergente con dos estudios sobre lectura y prosodia 
(Borzone de Manrique \& Signorini, 1991, 2000) en los cuales se observó que éste era un procedimiento frecuentemente encontrado en lectores de segundo o tercer grado y que permanecía en niños de sexto grado, en quienes se verificaban problemas de comprensión lectora.

En línea con estos trabajos se interpreta que la subvocalización durante la copia se asocia con una dificultad en el acceso a representaciones ortográficas de palabras. Cuando el escritor no puede instanciar eficientemente la palabra en su MO, recurre al análisis secuencial de los fonemas. Si bien este procedimiento desempeña un papel crucial en las primeras fases del aprendizaje tanto de la lectura (Signorini \& Piacente, 2001) como de la escritura (Borzone \& Diuk, 2001; Sánchez Abchi et al., 2009; Signorini \& Borzone de Manrique, 2003) su uso en situaciones de redacción puede entorpecer la ejecución simultánea de tareas de generación textual.

Los resultados obtenidos permiten inferir que el acceso poco fluido a representaciones de palabras influye en dos tareas de escritura de distinto nivel de complejidad. Estudios posteriores deberán indagar sistemáticamente los procesos de subvocalización y su relación con la complejidad de la tarea de escritura realizada.

La principal implicancia pedagógica de este trabajo es que la mejoría en el desempeño de los niños en tareas de escritura que supongan una diferente carga cognitiva depende de la consolidación del acceso fluido a representaciones ortográficas de palabras. En línea con esta conclusión cobra sentido la recomendación de Berninger y Richards (2002) quienes sugieren favorecer, en el primer ciclo, la realización de secuencias instruccionales breves y cercanas en el tiempo que aborden tanto la ejercitación del trazado manual, como el análisis de las palabras y las actividades de redacción. Estas sugerencias didácticas suponen ayudar a que la MO del niño pueda realizar eficientemente los 'malabares' (McCutchen, 1996) entre los distintos subprocesos involucrados en la escritura (motores, léxicos, discursivos). 
TABLA 1

MEDIAS Y DESVÍOS ESTÁNDAR DE FLUIDEZ DE COPIA (LETRAS POR MINUTO)

\begin{tabular}{|l|ll|lc|}
\hline & \multicolumn{2}{|c|}{ Presente estudio } & \multicolumn{2}{c|}{ Graham et al. (1998) } \\
& \multicolumn{2}{|c|}{$M$} & $D E$ & \multicolumn{2}{c|}{$M$} & $D E$ \\
\hline \multirow{2}{*}{ Total } & 47.35 & 18.25 & 47.3 & 13.40 \\
Niños & 41.49 & 16.24 & 44.8 & 12.57 \\
Niñas & 52.83 & 18.44 & 49.8 & 13.88 \\
Niños con sobre-edad & 51.3 & 22.7 & 84.74 & $18.96^{*}$ \\
& \multicolumn{2}{|c|}{} \\
\hline
\end{tabular}

*Las medidas de referencia para Niños y Niñas corresponden a sujetos de tercer grado. En cambio, para los Niños con sobre-edad fueron adoptadas las que corresponden a la edad promedio del presente estudio.

TABLA 2

MEDIAS DE FLUIDEZ DE COPIA DISCRIMINADAS POR ESCUELAS Y PROCEDENCIA SOCIAL

\begin{tabular}{|c|c|c|}
\hline Escuela & Procedencia social & Media de velocidad \\
\hline 1 & Media - alta & 50.07 \\
2 & Media - alta & 43.14 \\
3 & Media - baja & 55.13 \\
4 & Media - baja & 27.45 \\
5 & Baja & 57.48 \\
6 & Baja & 42.69 \\
\hline
\end{tabular}

FIGURA 1

FRAGMENTO DE UNA NARRACIÓN ESCRITA REDACTADA POR UNA NIÑA DE TERCER GRADO

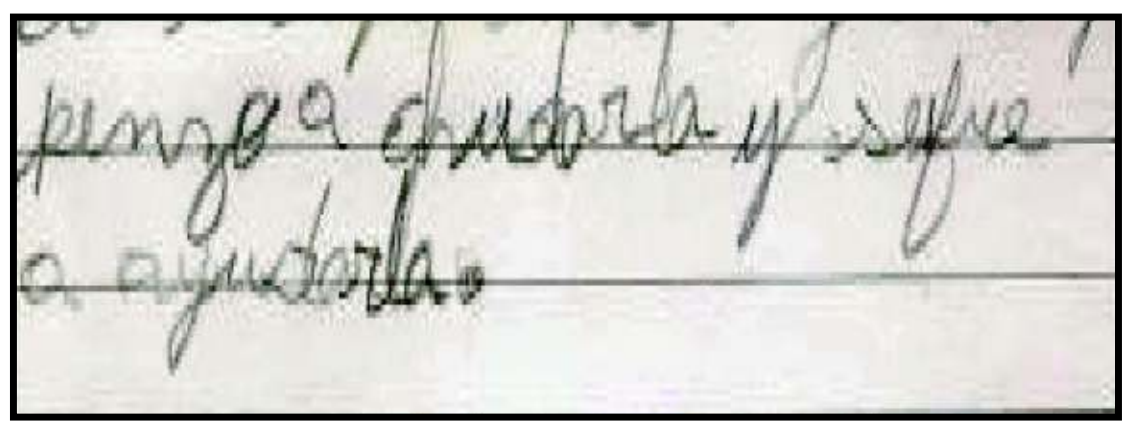




\section{REFERENCIAS BIBLIOGRÁFICAS}

Abadía de Quant, I. (2000). El español del nordeste [Spanish in Northeast Argentina]. En Fontanella de Weimberg (Comp.), El español de la Argentina y sus variedades regionales (pp. 101-137). Buenos Aires: Edicial.

Bereiter, C. \& Scardamalia, M. (1987). The psychology of writing composition. Hillsdale, NJ: Erlbaum.

Berninger, V., Abbott, R., Abbott, S., Graham, S. \& Richards, T. (2002). Writing and reading: Connections between language by hand and language by eye. Journal of Learning Disabilities, 35(1), 39-56. doi: 10.1177/00022219 40203500104.

Berninger, V. \& Fuller F. (1992). Gender differences in orthographic, verbal and compositional fluency: Implications for assessing writing disabilities in primary grade children. Journal School Psychological, 30, 363-382. doi:10.1016/0022-4405(92)90004-O.

Berninger, V. Garcia, N. \& Abbott, R. (2009). Multiple processes that matter in writing instruction and assessment. En G. Troia (Ed.), Instruction and assessment for struggling writers (pp. 15-50). NY: Guilford.

Berninger, V. \& Graham, S. (1998). Language by hand: A synthesis of a decade of research on handwriting. Handwriting Review 12, 11-25.

Berninger, V. \& Richards T. (2002). Brain literacy for educators and psychologists. California: Academic Press.

Berninger, V. \& Swanson, H. (1994). Modifying Hayes and Flower's model of skilled writing to explain beginning and developing writing. En E.C. Butterfield (Ed.), Children's writing: toward a process of development of skilled writing (pp. 57-81). Greenwich, CT: JAI.

Berninger, V. \& Winn,W. (2006). Implications of advancements in brain research and technology for writing development, writing in- struction, and educational evolution. En C. Mac Arthur, S. Graham, \& J. Fitzgerald (Eds.), Handbook of writing research (pp. 96114. NY: Guilford.

Borzone, A.M. \& Diuk, B. (2001). El aprendizaje de la escritura en español: Estudio comparativo entre niños de distinta procedencia social [Writing learning in Spanish. Comparative study between children different socio-economic level]. Interdisciplinaria, 18 (1), 35-63.

Borzone, A.M. \& Diuk, B. (2003). La escritura de textos en niños pequeños: Conocimiento ortográfico y producción textual [Text's writing of little children. Orthographic knowledge and text production]. Cultura y Educación, 15, 727.

Borzone, A.M., Rosemberg, C., Diuk, B., Silvestri A. \& Plana, D. (2004). Niños y maestros por el camino de la alfabetización [Children and teachers on the way of literacy]. Argentina: Red de Apoyo Escolar.

Borzone de Manrique, A. \& Signorini, A. (1991). La modalidad en lectura voz alta/silencio en la etapa inicial del aprendizaje [Oral and silent reading in the early stages of reading acquisition]. Revista Fonoaudiológica, 37(2), 2945.

Borzone de Manrique, A.M. \& Signorini, A. (2000). Lectura y prosodia: Una vía para el estudio del procesamiento cognitivo [Reading and prosody. A way to the study of cognitive processing]. Interdisciplinaria, 17(2), 95-117.

Bourdin, B. \& Fayol, M. (1994). Is written language production more difficult than oral language production? A working memory approach. International Journal of Psychology, 29, 591-620. doi:10.1080/0020759940 8248175.

Bourdin, B. \& Fayol, M. (2002). Even in adults, written production is still more costly than oral production. International Journal of Psychology, 37(4), 219-227. doi:10.1080/00207 590244000070 . 
Butterfield, E.C., Hacker, D.J. \& Albertson, L.R. (1996). Environmental, cognitive and metacognitive influences on text revision: Assessing the evidence. Educational Psychology Review, 8, 239-297. doi:10.1007/BF 01464075.

Condemarín, M. \& Chadwick, M. (1986). La escritura creativa y formal [Creative and formal writing]. Santiago de Chile: Andrés Bello.

Condemarín, M., Chadwick, M. \& Milicic, N. (1994). Madurez escolar [School maturity]. Santiago de Chile: Andrés Bello.

Cuetos, F., Ramos Sánchez, J. \& Ruano, L. (2002). PROESC Evaluación de los procesos de escritura [PROESC Assessment of writing processes]. Madrid: TEA Ediciones.

Defior Citoler, S., Fonseca, L. \& Gottheil, B. (2006). LEE. Lectura y escritura en Español [LEE. Reading and writing in Spanish]. Buenos Aires: Paidós.

Ehri, L. (1992). Reconceptualizing the development of sight word reading and its relationship to recoding. En P. Gought, L. Ehri \& R. Treiman (Eds.), Reading acquisition (pp. 107-143). Londres: Erlbaum.

Fonseca, L. (2009). LEE Test de Lectura y Escritura en Español: Su validez discriminante como instrumento de evaluación de las alteraciones de los procesos de lectura y escritura en niños [LEE reading and writing test in Spanish: Its discriminating validity as a tool for the assessment of reading and writing processes impairments]. Buenos Aires: FLACSO.

Grabowski, J. (2005). Speaking, writing, and memory span performance: Replicating the Bourdin and Fayol results on cognitive load in German children and adults. En L. Allal \& J. Dolz (Eds.), Proceedings Writing 2004 [CD]. Geneva (CH): Adcom Publications.

McArthur, C., Graham, S. \& Fitzgerald, J. (Eds.) (2006). Handbook of writing research. Mahwah. NJ: Erlbaum.
Graham, S. (1990). The role of production factors in learning disabled student's composition. Journal of Educational Psychology, 82, 781791.

Graham, S., Harris, R.K. \& Fink, B. (2000). Is handwriting causally related to learning to write? Treatment of handwriting problems in beginning writers. Journal of Educational Psychology, 92(4), 620-633. doi:10.1037/00 22-0663.92.4.620.

Graham, S. \& Weintraub, N. (1996). A review of handwriting research: Progress and prospects from 1980 to 1994. Educational Psychology Review, 8, 7-87. doi: 10.1007/BF01761831.

Graham, S., Weintraub, N. \& Berninger, V. (1998). The relationship between handwriting style and speed and legibility. Journal of Educational Research, 91(5), 290-296. doi:1 $0.1080 / 00220679809597556$.

Guinet, E. \& Kandel, S. (2010). Ductus: A software package for the study of handwriting production. Behavior Research Methods, 42(1), 326-332. doi:10.3758/BRM.42.1.326.

Hamstra-Bletz, L. \& Blote, A. (1990). Development of handwriting in primary school: A longitudinal study. Motor Skills, 70, 759-770. doi:10.2466/pms.1990.70.3.759.

Hamstra-Bletz, L. \& Blote, A. (1993). A longitudinal study on dysgraphic handwriting in primary school. Journal of Learning Disability, 26, 689-699. doi:10.1177/002221949 302601007.

Hayes, J. (2006). New directions in writing theory. En C. MacArthur, S. Graham \& J. Fitzgerald (Eds.), Handbook of writing research (pp. 28-40). NY: Guilford Press.

Jones, D. \& Christensen, C.A. (1999). The relationship between automaticity in handwriting and students' ability to generate written text. Journal of Educational Psychology, 91, 44-49. doi:10.1037/0022-0663. 91.1. 44.

Kellogg, R.T. (1996). A model of working memory in writing. En C.M. Levy \& S. 
Ransdell (Eds.), The science of writing: Theories, methods, individual differences and applications (pp. 57-72). Mahwah, NJ: Erlbaum.

Luria, A.R. (1973). The working brain. New York: Basic Books.

McCutchen, D. (1996). A capacity theory of writing: Working memory composition. Educational Psychology Review, 35, 13-23.

McCutchen, D. (2000). Knowledge, processing, and working memory: Implications for a theory of writing. Educational Psychologist, 35, 1323. doi: 10.1207/S15326985EP35013.

MECyT (2006). NAP Cuaderno para el aula 3 [NAP Workbook for classroom 3]. Buenos Aires: CFCE.

Medwell, J. \& Wray, D. (2008). Handwriting - A forgotten language skill? Language and Education, 22(1), 34-47. doi:10.2167/le722.0.

Olive, M., Beauvais, C. \& Beauvais, L. (2009). Children's cognitive effort and fluency in writing: Effects of genre and of handwriting automatisation. Learning and Instruction, 19 (4), 299-308. doi:10.1016/j.learninstruc.200 8.05.005

Overvelde, A. \& Husltijn, W. (2011). Handwriting development in grade 2 and grade 3 primary school children with normal, at risk, or dysgraphic characteristics. Research in Developmental Disabilities, 32, 540-548. doi: 10.10 16/j.ridd.2010.12.027.

Perfetti, C. (1992). The representation problem in reading acquisition. En P. Gough, L. Ehri \& R. Treiman (Eds.), Reading acquisition (pp. 145174). Hillsdale NJ: Erlbaum.

Pollock, N., Lockhart, J., Blowes, B., Semple, K., Webster, M., Farhat, L. et al. (2009). Handwriting assessment protocol (2da. ed.). Can Child, McMaster University.

Rodríguez, C., González, P., Álvarez, L., Cerezo, R., García, J., González, J. et al. (2010). Un análisis de los modelos teóricos actuales de escritura y su relación con el trastorno de déficit de atención e hiperactividad (TDAH) [An analysis of theoretical models of writing and their relation with Attention Deficit and Hyperactivity Disorder (ADHD)]. Papeles del Psicólogo, 31(2), 211-220.

Rosemblum, P., Weiss, P. \& Parush, S. (2004). Handwriting evaluation for developmental dysgraphia: Process versus product. Reading and Writing, 17(5), 433-458. doi: 10.1023/ B: READ.0000044596.91833.55.

Rosenblum, S., Parush, S. \& Weiss, P. (2001). Temporal measures of poor and proficient handwriters. En R.G.J. Meulenbroek \& B. Steenbergen (Eds.) Proceedings of the Tenth Biennial Conference of the International Graphonomics Society (pp. 119-125). The Netherlands: University of Nijmegen.

Sánchez Abchi, V., Borzone, A. \& Diuk, B. (2007). La escritura de textos en niños pequeños: Relación entre la transcripción y la composición [Text writing in small children: Relationship between transcription and composition]. Revista Universitas Psychologica, 6 (3), 559-569.

Sánchez Abchi, V., Diuk, B., Borzone, A. \& Ferroni, M. (2009). El desarrollo de la escritura de palabras en Español: Interacción entre el conocimiento fonológico y ortográfico [Spelling development in Spanish: Interaction between phonological and orthographic knowledge]. Interdisciplinaria, 26(1), 95-119.

Signorini, A. \& Borzone de Manrique, A. (2003). Estrategias fonológicas y aprendizaje de la lectura y la escritura [Learning to read and spell in Spanish. The prevalence of phonological strategies]. Interdisciplinaria, 20(1), 5- 30.

Signorini, A. \& Piacente, T. (2001). Adquisición de la lectura en español: Habilidades de procesamiento de palabras en lectores iniciales [Reading acquisition in Spanish. Words processing skills in beginner readers]. Revista IRICE, 15, 5-29.

Toro, J., Cervera, M. \& Urío, C. (2000). EMLE Escalas Magallanes de Lectura y Escritura 
TALE-2000. Manual de referencia [EMLE Reading and Writing Magallanes Scales. Reference guide]. Bizkaia: COHS.

Trabasso, T. \& Rodkin, P. (1994). Knowledge of goal/plans: A conceptual basis for narrating Frog, where are you? En R. Berman \& D. Slobin (Eds.), Relating events in narrative: $A$ crosslinguistic developmental study (pp. 85106). NJ: Erlbaum.

Troia, G. (Ed.) (2009). Instruction and assessment for struggling writers. NY: Guilford.

Tuana, E. (1980). Estudio comparativo de la ortografía de los niños normales y niños con dificultades de aprendizaje [Comparative study of orthography in normal children and children with learning disabilities]. Lectura y Vida, 1(1), 16-21.
Yausaz, F. (2005). El desarrollo de habilidades de escritura de textos en niños pequeños: El impacto de un taller escolar de composición [Development of composition skills in young children: Impact of a school composition workshop]. Cultura y Educación, 17(4), 349-360. doi: 10.1174/113564005775133784.

Yausaz, F. (2011). Escribi como puedas. Estudios sobre la composición escrita al finalizar tercer grado [Write you can. Studies on written composition at the end of third grade]. Tesis Doctoral no publicada. Facultad de Humanidades y Artes de la Universidad Nacional del Nordeste. Resistencia. Argentina.

Instituto Superior San José Corrientes República Argentina

Fecha de recepción: 1 de agosto de 2011 Fecha de aceptación: 15 de octubre de 2012 